\title{
Theoretical Study of One-dimensional Chains of Metal Atoms in Nanotubes
}

\author{
Angel Rubiot, Yoshiyuki Miyamotol, X. Blase, Marvin L. Cohen, and Steven G. Louie \\ Department of Physics, University of California at Berkeley, Berkeley, California 94720 and \\ Materials Sciences Division, Lawrence Berkeley Laboratory, Berkeley, California 94720
}

(October 24, 2018)

\begin{abstract}
Using first-principles total-energy pseudopotential calculations, we have studied the properties of chains of potassium and aluminum in nanotubes. For BN tubes, there is little interaction between the metal chains and the tubes, and the conductivity of these tubes is through carriers located at the inner part of the tube. In contrast, for small radius carbon nanotubes, there are two types of interactions: charge-transfer (dominant for alkali atoms) leading to strong ionic cohesion, and hybridization (for multivalent metal atoms) resulting in a smaller cohesion. For Al-atomic chains in carbon tubes, we show that both effects contribute. New electronic properties related to these confined atomic chains of metal are analyzed.
\end{abstract}

PACS numbers: 71.25.Tn, 36.20.Kd

Typeset using REVTEX 
Quasi-one-dimensional metals and conducting nanowires are of great interest both from a fundamental point of view and for possible applications. Here we explore theoretical models for examining the structural and transport properties of these systems [1]. Tubule forms of graphitic carbon [2] with their expected interesting electronic and mechanical properties [3] 8 are ideal candidates as hosts for one dimensional metal systems. Carbon tubes have been made which are microns in length [2,07-10] and have diameters ranging from larger than $100 \AA$ for multi-wall tubes down to less than $10 \AA$ for single-wall tubes [10,11]. Important effects in the conductivity as a function of length and diameter of encapsulated metallic nanowires are expected and confinement might induce new metallic phases. For small diameter tubes, the captured metal atoms inside can form an atomic linear-chain [4. This would provide a new means for producing ideal one-dimensional metallic chains. The metal capillarity and doping of tubes also raise the possibility of changing the electronic structure of the tube itself by either charge-transfer or hybridization. Just as in the case of alkali doped $\mathrm{C}_{60}$, we expect curvature effects to alter electron-phonon and superconducting pairing interactions from those of intercalated graphite [12].

At present, we know of no evidence of alkali intercalation into nanotubes experimentally which is in contrast to the results for graphitic intercalation compounds (GIC's) [13]. Most of the experiments on metal-atom intercalation have been done on multi-wall tubes with large diameter sizes (more than $100 \AA$ diameter) [5 0,9,14]. Transition metals inside the tubes were studied [7], and it was found that the formation of continuous nanowires was connected with the existence of an incomplete electronic shell in the most stable ion configuration of the metal. However, in most cases, the filling material was a crystalline metallic carbide. Lead, bismuth and manganese incorporation has also been reported [5,9]. A more detailed study [6] has proposed surface tension of the liquid phase of the metals as a key factor in determining whether capillary action (wetting) occurs. The experimental surface tension threshold found for wetting is near $190 \mathrm{mN} / \mathrm{m}$. Thus, in this picture, fillings using metals with larger surface tension require external pressure to be experimentally realizable, and it is expected that typical metals will not be drawn into tube cavities by capillary action. 
On the other hand, a strong ionic cohesion for K inside subnanometer-size carbon tube was theoretically predicted [4]. The assumed classical theory of wetting was therefore concluded to be inappropriate for this tube size, and incorporation of other metal-atoms should be possible.

Based on the similarities among graphite and hexagonal boron-nitride compounds, we have predicted that $\mathrm{BN}$ and $\mathrm{B}_{x} \mathrm{C}_{y} \mathrm{~N}_{z}$ will form stable tubes [15,16]. This was recently proven experimentally by electric arc-discharge synthesis [17] as well as by laser-driven gas-phase chemical reaction synthesis [18]. The electronic properties of this new class of nanotubes are quite different from their carbon counterpart. Namely, the BN nanotubes are stable wide band-gap semiconductors $\left(\mathrm{E}_{\mathrm{g}} \sim 5.5 \mathrm{eV}\right)$ independent of helicity, diameter of the tube, or whether the tube is single wall or multi-wall. Furthermore, the bottom of the conduction band is a nearly free electron (NFE) like-state that derives its character from the weakely bound states of a $\mathrm{BN}$ sheet in a band-folding picture [19. Considering that insulators are much less polarizable than metals and semimetals, it is expected that the potential experienced by an electron in the internal vacuum region close to the $\mathrm{BN}$ tube surface will be less binding than that for graphite. Therefore, we expect BN to behave like an ideal non-interacting host for the metal atoms inside. We note here that other composition $\mathrm{B}-\mathrm{C}-\mathrm{N}$ tubes have also been proposed $\left(\mathrm{BC}_{2} \mathrm{~N}\right.$ and $\left.\mathrm{BC}_{3}\right)$ as stable [16] and observed experimentally [20]. These systems have potential technological applications and have very interesting electronic properties which can be generally explained by rolling the corresponding planar sheets. However, we expect that these B-C-N tubes would not serve as non-interacting hosts since their band-gaps are small.

The purpose of the present study is to examine two particular cases of intercalation with metal atoms of different chemical valence: $\mathrm{K}$ and $\mathrm{Al}$ atoms. We compare and contrast the results for BN tubes with those for carbon nanotubes. The known alkali GIC's [13] and the intercalation of K-atoms in hexagonal BN [21] supports the possibility of incorporation of this metal into small radius nanotubes. However, there are no reports for Al GIC's and Al intercalation into hexagonal BN to our knowledge, so no comparison can be made. We show 
that $\mathrm{BN}$ tubes behave as non-interacting confining hosts for metallic chains made of $\mathrm{K}$ (or other alkali atoms) and Al. This is in contrast with metal atoms inside carbon nanotubes where charge-transfer and hybridization effects are obtained. In all cases, we expect that reactions in the gas phase would favor the intercalation of these metallic atoms in both carbon and BN nanotubes. We conclude that the thin tubes react with metals similarly to the process of intercalation of the planar graphitic sheets.

Total-energy pseudopotential band-structure calculations are done for the metal and tube systems within the framework of the local density approximation (LDA) using a planewave basis set with a cutoff energy of $36 \mathrm{Ry}$. The Kleinman-Bylander ab-initio pseudopotential scheme [22] including core-corrections for the exchange-correlation energy [23] is used. The core correction is necessary to reproduce the structural properties of bulk K. The calculations were performed in a supercell geometry with a $5.5 \AA$ distance between the walls of neighboring tubes. This distance is large enough to ensure that tube-tube interactions are negligible. We use two k-points in the one dimensional irreducible Brillouin zone to get well converged total energies and electronic states.

We first perform a structural minimization for the free standing linear chain of $\mathrm{K}$ and $\mathrm{Al}$ atoms. The obtained bond length of the $\mathrm{K}$ chain is $4.08 \AA$ and the $\mathrm{Al}$ chain $2.38 \AA$, as compared to the calculated values of $4.38 \AA$ for bulk bcc $\mathrm{K}$ and $2.84 \AA$ for bulk fcc Al. The smaller bond-length can be understood in terms of a reduction of the coordination number going from the bulk system to the linear chain [24]. In the case of a $K$ chain, a small energy lowering (below $0.5 \mathrm{~K}$ ) [4] was found by dimerization of the $\mathrm{K}$ atoms. In contrast, an energy lowering of the $\mathrm{Al}$ chain from dimerization was not found in the present calculations. These results however do not rule out a Peierls transition for $\mathrm{K}$ and $\mathrm{Al}$ chains. Since the energy gain is extremely small, the calculations require a large number of sampling points in momentum space. According to these results, we believe that Peierls distortion would not be important in practical situations. We have estimated the cohesive energy to be $0.47 \mathrm{eV} /$ atom and $1.23 \mathrm{eV} /$ atom for the $\mathrm{K}$ and $\mathrm{Al}$ chains respectively, as compared to the experimental values of $0.94 \mathrm{eV} /$ atom and $3.34 \mathrm{eV} /$ atom for bulk $\mathrm{K}$ and $\mathrm{Al}$ respectively [25]. For the $\mathrm{Al}$ chain, 
the large difference in cohesive energy with respect to the bulk metal can be related to the incomplete formation of bulk sp-delocalized bands in the one dimensional chain. Similar effects are expected to happen for the electronic structure when the Al chain is incorporated into a BN nanotube.

In order to reduce the computational effort, we have assumed that the linear chain and the nanotube constitute commensurate phases because the change in the binding energy of the linear chain in going from the theoretical bond length to the well-matched tube lattice constant is small [26]. Thus we set the metal chain bond length to be the same as the periodic distance along the tube axis in all the calculations reported here. We note that the $\mathrm{K}$ chain is well suited to be incorporated in $(n, 0)$ single unit cell tubes or double unit cell $(n, n)$ nanotubes, while the $\mathrm{Al}$ chain fits well in the $(n, n)$ single unit cell nanotubes [27. The index notation for the tubes used here is the same as that given in Ref. [3]. Furthermore, the metal atoms are assumed to be linearly aligned at the tube center. This is not a severe approximation since for the diameter of the tubes studied, the metallic linear chain is in the only possible arrangement because of the geometrical restrictions for the covalent and metallic radii of the atoms [24].

In Ref. [4] there is a discussion of the important role of having small diameter carbon tubes (diameters close to the GIC's interlayer distance) for maximizing the heat of formation for incorporating $\mathrm{K}$ atoms $(\sim 1 \mathrm{eV} / \mathrm{K}$-atom). The heat of formation is obtained by subtracting the total energy of the doped tube from the sum of the total energies of separated systems of bulk metal and a pure tube. This subnanometer diameter tubes do not obey the classical picture of wetting and capillarity. We here note that non-intercalation of $\mathrm{Al}$ atoms is expected for very large diameter tubes because liquid Al has a much larger surface tension 25] than the threshold for wetting [6]. For the intercalation of $\mathrm{Al}$ in a $\mathrm{C}(4,4)$-tube, we find that the heat of formation is negative, in contrast to K. However, there is a gain in energy of $0.14 \mathrm{eV} /$ atom comparing the energy of a tube with a linear chain inside to that with the chain separated. We thus expect intercalation of $\mathrm{Al}$ in the gas phase could be experimentally realizable under pressure for carbon tube bundles. The main reason for the negative heat of 
formation is that the coordination number for $\mathrm{Al}$ is not large enough to form the $s p$-metallic band of bulk Al. Hence we expect that $\mathrm{Al}$ is likely to form larger diameter nanowires (with a tendency to fcc coordination) when the tube diameter is larger but still in the nanometer regime. In Fig. (1) the band structure of $\mathrm{Al}$ inside a $\mathrm{C}(4,4)$ nanotube is compared with that of an undoped tube. In Fig. (2), a band structure of a free standing $\mathrm{Al}$ chain is shown for the comparison. Upon $\mathrm{Al}$ incorporation, the Fermi level is shifted upward corresponding to one electron transfer per metal atom as in the case of K-intercalation. On the other hand, some hybridization between the Al-s states with the tube wall is observed. This Al-state is indicated in Fig. (1). The $\mathrm{Al} \mathrm{p}_{z}$-states and $\mathrm{p}_{x y}$-states are above the Fermi-level and also hybridize with the conduction tube states.

To gain insight into the physics of intercalation of $\mathrm{K}$ atoms into $\mathrm{BN}$ tubes, we first performed some calculations for the hexagonal planar phase. Hexagonal BN is an indirect wide-gap semiconductor with a NFE state which lies close to the bottom of the conduction band [19]. This NFE state is the one that interacts the most with the $\mathrm{K} s$ states. The position of the $\mathrm{K}$-derived level, around $3 \mathrm{eV}$ above the $\mathrm{BN}$ valence band top, is consistent with photoluminescence experiments [21]. We remark that the band structure of a single $\mathrm{BN}$-sheet is also a wide band gap insulator but the NFE state now becomes the bottom of the conduction band. According to our calculation, the wetting of the planar BN sheet by a K-monolayer is an exothermic reaction $(\sim 0.5 \mathrm{eV} / \mathrm{K}$-atom $)$ with a $2 \times 2$ unit cell. In a manner similar to the adsorption of $\mathrm{K}$ layers on graphite [28], multiple-layers are not expected to be adsorbed on the BN sheet. These results support the possible intercalation of $\mathrm{K}$ atoms in small radius $\mathrm{BN}$ nanotubes. In the three cases studied here: $\mathrm{K}$ atoms inside $\mathrm{BN}(4,4)$ and $\mathrm{BN}(8,0)$, and $\mathrm{Al}$ atoms in $\mathrm{BN}(4,4)$ the difference in the binding energy of the two systems (energy of the tube with metal outside minus the one with metal inside) is positive but smaller than the value for $\mathrm{Al}$ in carbon nanotubes mainly due to the negligible charge-transfer from the chain to the $\mathrm{BN}$-wall. This is an indication of weak interaction between the metal-chain and the BN-tube. Intercalation however could still be possible while growing the nanotube in a vapor phase of the metallic element. 
The band structures of $\mathrm{K}$ and $\mathrm{Al}$ chains in $\mathrm{BN}(4,4)$ tubes are depicted in Fig. (3). The NFE state interacts with the K chain $s$ states, keeping the wavefunction of the occupied carrier states located in the interior of the tube on the $\mathrm{K}$ chain (see a contour map of charge density for the state $\alpha$ at the bottom of Fig. (3)). This is different from K in carbon nanotubes, in which the $\mathrm{K}$ chain $s$ states and the carbon NFE state also interact but the corresponding wavefunctions are unoccupied and the $\mathrm{K}$ electrons are donated to the graphitic wall. We check that these results are independent of the diameter and helicity of the $\mathrm{BN}$ tube by doing the calculation for $\mathrm{K}$ in $\mathrm{BN}(8,0)$. The same picture is obtained with hybridization among the tube NFE-band and the K-s derived band forming occupied states with wavefunction inside the tube. The band structures of the multiwall BN-nanotubes do not change significantly from those of the single wall tubes, hence we expect the intercalation to be the same as for the single wall tubes.

The band structure for an $\mathrm{Al}$ chain in a $\mathrm{BN}(4,4)$ tube can be understood by the direct addition of the two band structures of the isolated systems (BN tube plus free $\mathrm{Al}$ chain). Electronic states in the gap at $\mathrm{X}$ are clearly seen as being derived from $\mathrm{Al} s$ and $p_{z}$ linear chain states, (see Fig. (2)). This is different from the carbon nanotubes where the $\mathrm{Al} p_{z}$ states are located just close to the Fermi level. The $\mathrm{Al} s$ state at $\mathrm{X}$ is displayed in the bottom part of Fig. (3). Also, plotted is one of the states at $\Gamma$ derived from the interaction of the NFE-state with the $\mathrm{Al} p_{x y}$ states $\left(\beta_{1}\right.$ and $\beta_{2}$ states in the figure which have very similar charge density profiles). We note that all of these states have charge density concentrated in the inner part of the tube. Therefore, the conductivity of these doped-tubes will be controlled by carriers in the inside of the tubes whereas, in carbon nanotubes, carriers on the tube wall dominate. When considering the rigid sliding motion of the inner metal chain, we find that the change in energy as a function of position is small and comparable to the one given for $\mathrm{K}$ in carbon nanotubes 团.

In conclusion, we have shown that boron-nitride nanotubes may be good hosts for the incorporation of metal nanowires. This together with the elastic properties and high thermal conductivity of the BN-matrix suggest that these systems may have technological applica- 
tions. Similar studies on carbon nanotubes show both charge-transfer and hybridization mechanisms are possible for the formation of the metal/tube system. The conductivity of the intercalated tubes will be dominated by carriers in the inner region of the tube for boron-nitride tubes and on the tube wall for carbon nanotubes. The transition between macroscopic behavior controlled by surface tension and the microscopic level incorporation studied here is still an open question. Noble-metal atoms such as Ag and Au could also be incorporated in these tubes forming continuous nanowires. Quantized conductance versus localization effect as a function of length has been reported for thin Au-wires [1]. Similar or more striking effects may be observed in a one-dimensional metal chain inside a tube.

Acknowledgements: This work was supported by National Science Foundation Grant No. DMR-9520554 and by the Director Office of Energy Research, Office of Basic Energy Sciences, Materials Sciences Division of the U.S. Department of Energy under Contact No. DE-AC03-76SF00098. The computations were done on the CRAY-C90 computer at the San Diego Supercomputer Center. X.B. gratefully acknowledges support from the FranceBerkeley fund. 


\section{REFERENCES}

$\dagger \quad$ Permanent address, Departamento de Física Teórica. Universidad de Valladolid, E47011 Valladolid, SPAIN

* Permanent address, Fundamental Research Laboratories, NEC Corporation, 34 Miyukigaoka, Tsukuba 305, JAPAN

[1] J.I. Pascual, J. Méndez, J. Gómez-Herrero, A.M. Baró, N. Garcia, U. Ladman, W.D. Luedtke, E.N. Bogachek and H.P. Cheng, Science 267, 1793 (1995).

[2] S. Iijima, Nature 354, 56 (1991).

[3] N. Hamada, S. Sawada, and A. Oshiyama, Phys. Rev. Lett. 68, 1579 (1992); R. Saito, M. Fujita, G. Dresselhaus, and M.S. Dresselhaus, Appl. Phys. Lett 60, 2204 (1992); X. Blase, L.X. Benedict, E.L. Shirley and S.G. Louie, Phys. Rev. Lett. 72, 1878 (1994).

[4] Y. Miyamoto, A. Rubio, X. Blase, M.L. Cohen and S.G. Louie, Phys. Rev. Lett. 74, 2993 (1995).

[5] P.M. Ajayan and S. Iijima, Nature 361, 333 (1993).

[6] E. Dujardin, T.W. Ebbessen, H. Hiura, and K. Tanigaki, Science 265, 1850(1994).

[7] C. Guerret-Plécourt, Y. Lebouas, A. Loisea and H. Pascard, Nature 372, 761 (1994).

[8] W.A. de Heer, W.S. Bacsa, A. Chatelain, T. Gerfin, R. Humphrey-Baker, L. Forro and D. Ugarte, Science 268, 845 (1995).

[9] P.M. Ajayan, C. Colliex, J.M. Lambert, P. Bernier, L. Barbedette, M. Tencé and O. Stephan, Phys. Rev. Lett. 72, 1722 (1994).

[10] S. Iijima and T. Ichihashi, Nature 363, 603 (1993).

[11] D.S. Bethune, C.H. Klang, M.S. de Vrles, G. Gorman, R. Savoy, J. Vazquez, and R. Beyers, Nature 363, 605 (1993). 
[12] L.X. Benedict, V.H. Crespi, S.G. Louie and M.L. Cohen (preprint)

[13] see for example, M.S. Dresselhaus and G. Dresselhaus, Advance in Physics, 30, 139 (1981), and references therein.

[14] K. Tanaka, T. Sato, T. Yamabe, K. Okahara, K. Uchida, M. Yumura, H. Niino, S. Ohshima, Y. Kuriki, K. Yase, and F. Ikazaki, Chem. Phys. Lett. 223, 65 (1994).

[15] A. Rubio, J.L. Corkill and M.L. Cohen, Phys. Rev. B 49, 5081 (1994); X. Blase, A. Rubio, S.G. Louie and M.L. Cohen, Europhys. Lett. 28, 335 (1994).

[16] Y. Miyamoto, A. Rubio, S.G. Louie, and M.L. Cohen, Phys.Rev.B 50, 4976 (1994); ibid. 5018360 (1994).

[17] N.G. Chopra, J. Luyken, K. Cherrey, V.H. Crespi, M.L. Cohen, S.G. Louie and A. Zettl, Science (in press).

[18] L. Boulanger, B. Andriot, M. Cauchetier and F. Willaime, Chem. Phys. Letts. 234, 227 (1995)

[19] X. Blase, A. Rubio, S.G. Louie and M.L. Cohen, Phys. Rev. B 51, 6868 (1995); A. Castellani, M. Posternack, A. Baldereschi, H.J.F. Jansen and A.J. Freeman, Phys.Rev.B 32, 6997 (1985).

[20] Z. Weng-Sieh, K. Cherrey, N.G. Chopra, X. Blase, Y. Miyamotot, A. Rubio, M.L. Cohen, S.G. Louie, A. Zettl and R. Gronsky, Phys.Rev B 51, 11229 (1995); O. Stephan, P.M. Ajayan, C. Colliex, Ph. Redlich, J.M. Lambert, P. Bernier and P. Lefin, Science 266, 1683 (1994).

[21] G.L. Doll, J.S. Speck, G. Dresselhaus, M.S. Dresselhaus, K. Nakamura, and S.I. Tanuma, J. Apply. Phys. 66, 2554 (1989).

[22] L. Kleinman and D.M. Bylander, Phys. Rev. Lett. 48, 1425 (1982).

[23] S.G. Louie, S. Froyen, and M.L. Cohen, Phys. Rev. B 26 , 1738 (1982). 
[24] L. Pauling, The nature of the chemical bond (Ithaca, New York, Cornell University Press, 1948), see chapter XI.

[25] Handbook of Chemistry and Physics, edited by R.C. Weast. (The Chemical Rubber Co., Cleveland, Ohio, 1972-1973.). The surface tension for liquid Al ranges from 400 to 900 $\mathrm{mN} / \mathrm{m}$ depending on the temperature and surrounding gas.

[26] This change in binding is of $0.011 \mathrm{eV} /$ atom and $0.04 \mathrm{eV} /$ atom for $\mathrm{K}$ - and Al-chains, respectively.

[27] The lattice constant for $(n, 0)$ tubes is $4.29 \stackrel{\circ}{A}$ and $4.32 \stackrel{\circ}{A}$ for carbon and BN, respectively. Meanwhile, the lattice constant for $(n, n)$ tubes is $2.46 \AA$ for $\mathrm{C}$ and $2.48 \AA$ for $\mathrm{BN}$.

[28] Z.Y. Li, K.M. Hock, and P.E. Palmer, Phys. Rev. Lett. 67, 1562 (1991). 


\section{FIGURES}

FIG. 1. Calculated band structures: (a) $(4,4)$ carbon tube and (b) Al-intercalated $(4,4)$ carbon tube. The location of the Fermi level $E_{F}$ as well as the Al-chain derived states are indicated.

FIG. 2. Band structure of a free standing Al linear chain.

FIG. 3. Top, calculated band structures: (a) K-intercalated $\mathrm{BN}(4,4)$ tube, pure $\mathrm{BN}(4,4)$ tube in a (b) single and (c) double unit cell, and (d) Al-intercalated $\mathrm{BN}(4,4)$ tube. We include (b) and (c) (related by folding) to allow comparison with the corresponding doped tubes. We note that the Brillouin zone of the $\mathrm{K}$ case is half that of Al. The maxima of the valence bands of the undoped tubes is set as the energy origin. The location of the Fermi level $E_{F}$ is indicated. The NFE state and the states that derive from its interaction with the metallic-chain states are

indicated and plotted in the bottom panel (states $\alpha, \beta_{1}$, similarly for $\beta_{2}$ ). Also the $\mathrm{Al} s$ - and $p_{z}$-states are indicated and the $\mathrm{Al} s$ charge density is plotted. 

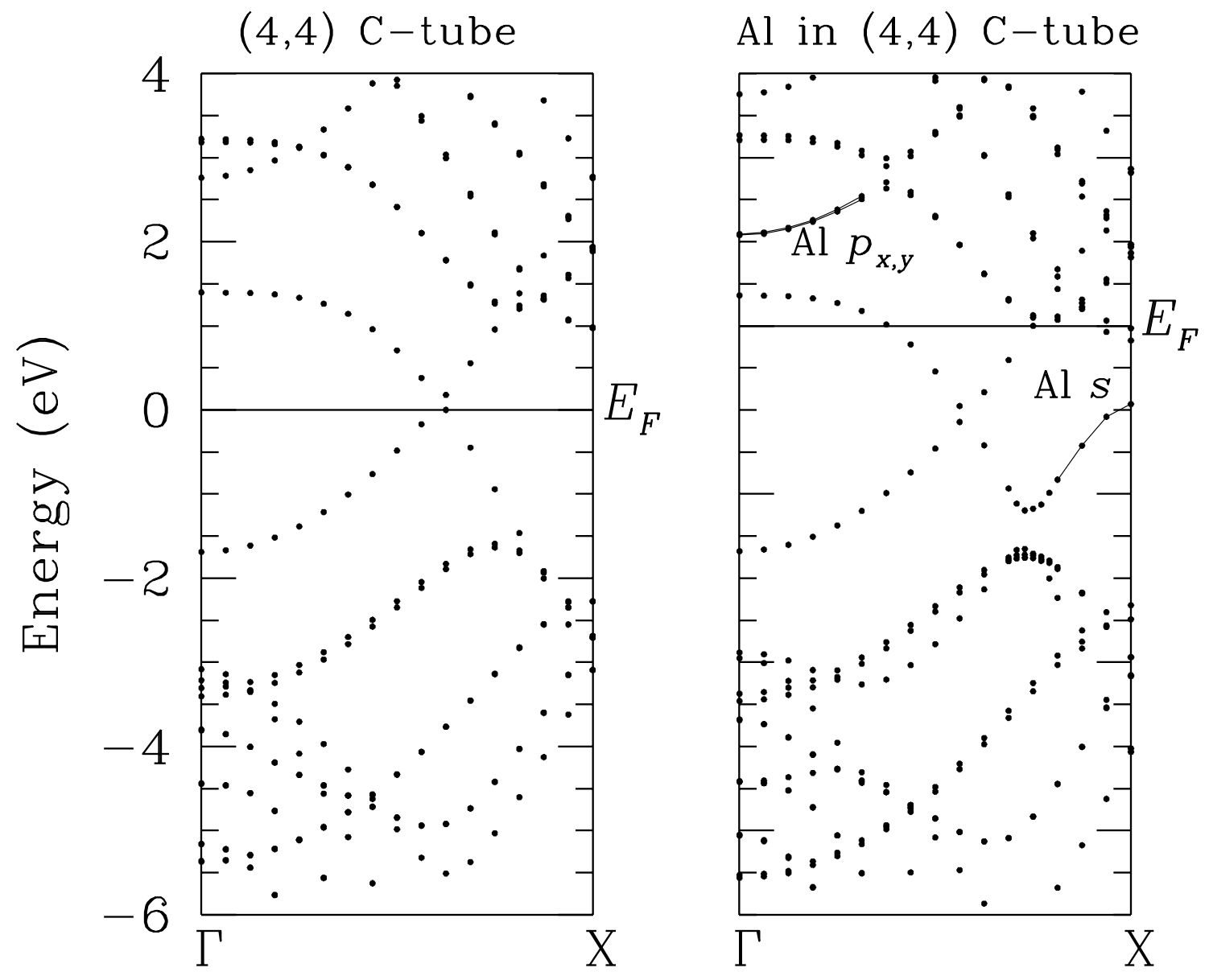

Fig.(1) Rubio et al. 


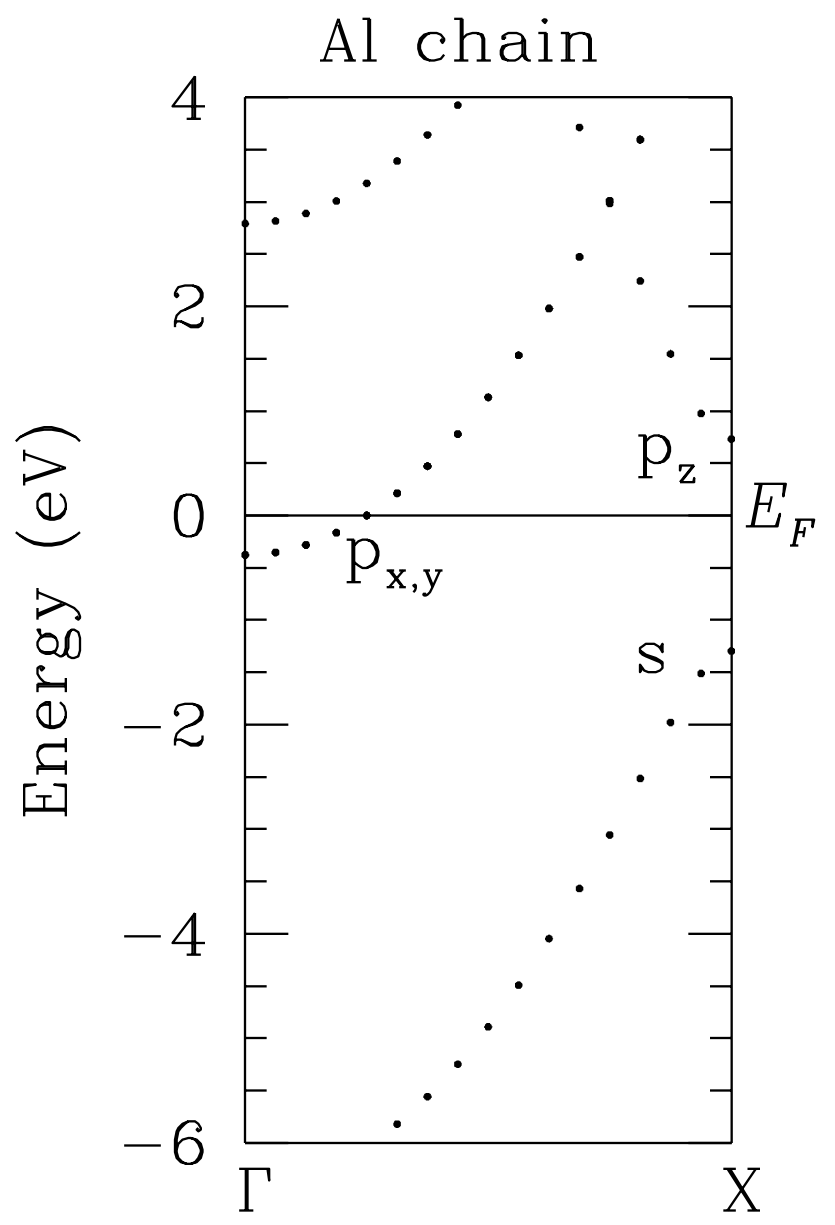

Fig.(2) Rubio et al. 

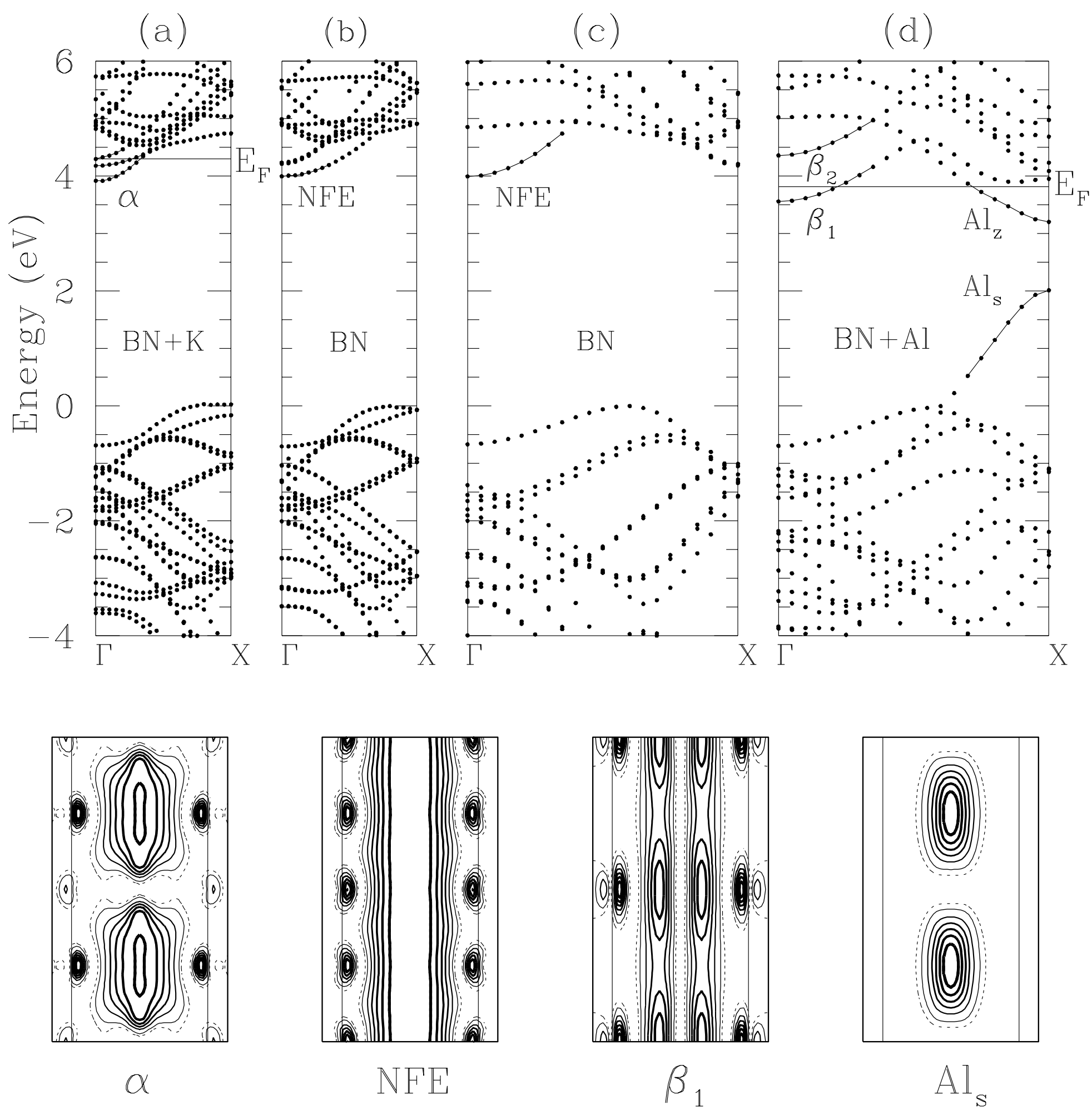